\title{
ON GENERALIZED JACOBIANS
}

\author{
BY
}

T. RADO AND P. V. REICHELDERFER

\section{INTRODUCTION}

1.1. The title of this paper refers to the Jacobians introduced by the writers $[2, \S 1.30 ; 6, I V .3]\left({ }^{1}\right)$ and used by them extensively in their work on the theory of the Lebesgue area of surfaces. During the war years Cesari in Italy developed a theory based on similar concepts $[3,4]$. Relationships between these two theories have been investigated in some detail in [5] and have been further clarified by Cesari in his invited address on Area and representation of surfaces, presented at the annual meeting of the American Mathematical Society in December 1948. This address is to appear in the Bulletin of the American Mathematical Society. In the sequel references to previous literature will be made only in connection with technical matters. The reader interested in historical background is referred to the address of Cesari and to the Colloquium publication [6].

1.2. The principal issue to be studied in this paper may be described as follows. Let $\Re$ be a bounded simply connected Jordan region in a Euclidean $u v$-plane, and let $x_{i}(u, v), i=1,2,3$, be continuous functions in $\Re$. If $x_{1}, x_{2}, x_{3}$ are Cartesian coordinates in Euclidean three space $E_{3}$, then the equations $x_{i}=x_{i}(u, v),(u, v) \in \Re$, for $i=1,2,3$ determine an $F$-surface $S$ of the type of the 2-cell [6, II.3.44]. If the Lebesgue area of $S$ is finite then there exist generalized Jacobians almost everywhere in the interior $\Re^{0}$ of $\Re[6$, V.2.61]. Now let $\bar{x}_{1}, \bar{x}_{2}, \bar{x}_{3}$ be Cartesian coordinates in $E_{3}$ related to $x_{1}, x_{2}, x_{3}$ by transformation formulas $\bar{x}_{i}=a_{i j} x_{j}$ for $i=1,2,3$, where repeated index $j$ indicates a summation over the range of $j$, and $\left\|a_{i j}\right\|$ is a matrix of real numbers which is normal, orthogonal, and has a determinant equal to +1 . The generalized Jacobians relative to these two systems of coordinates are related to each other almost everywhere in $\Re^{0}$ by the linear transformation with the matrix $\left\|a_{i j}\right\|$ $[10, \S 4]$. However, the exceptional set of points in $\Re^{0}$ of measure zero at which no relationship between the Jacobians is available depends upon the frames of reference $x_{1}, x_{2}, x_{3}$ and $\bar{x}_{1}, \bar{x}_{2}, \bar{x}_{3}$ in $E_{3}$. There arises the question as to whether the transformation formulas for the generalized Jacobians actually hold "uniformly almost everywhere" in $\Re^{0}$ in the sense that there is a fixed set of measure zero in $\Re^{0}$ such that at every point in $\Re^{0}$ not in this set, for every choice of the frames of reference $x_{1}, x_{2}, x_{3}$ and $\bar{x}_{1}, \bar{x}_{2}, \bar{x}_{3}$, the generalized Jacobians are defined and related by the same law which relates the coordinates

Presented to the Society, September 1, 1949; received by the editors April 28, 1949.

(1) Numbers in brackets refer to the bibliography at the end of the paper. 
in the frames of references. In view of the linear character of the transformation formulas this question may seem trivial, yet we are unable to give a final answer to the question in the form just stated. On the other hand, we have succeeded in answering a series of related questions concerning the "uniformly almost everywhere" validity of various fundamental relationships amongst generalized Jacobians. Our main results are summarized in the theorem in $\$ 3.14$. The fourth part of this paper contains an application to the ordinary Jacobians.

1.3. While the theory of the Lebesgue area is now practically complete for surfaces of the type of the 2-cell, the extension of this theory to higher dimensions has been initiated only recently by Federer [7]. It appears that various alternative definitions of the fundamental concepts, which are fully interchangeable in the 2-cell case, become essentially different in higher dimensions. The case of the essential multiplicity function $\kappa(6, I V .1)$ is a significant example. In the 2-cell case there are at least four alternative definitions for $\kappa$, which were denoted by $\psi, \psi^{*}, \Psi, \Psi^{*}$ in $(5,1.1 .11)$. Of these, $\psi^{*}$ coincides with the original $\kappa, \Psi$ is the multiplicity function used by Cesari (see $\S 1.1$ ), while $\psi$ and $\Psi^{*}$ were introduced essentially as auxiliary concepts. It was shown that these four functions differ from each other only on a countable set of points [5, I.4.6], and hence are fully interchangeable. On the other hand, the work of Federer indicates that in higher dimensions, the function defined analogously to $\Psi^{*}$ may be the most suitable choice. Since ${ }^{*}$ is used in the sequel for a specific purpose, let us use $\mathrm{K}$ as a more convenient notation for $\Psi^{*}$. For the purpose of extending the theory to higher dimensions, the following comment may be relevant. In the 2-cell case, essential use is made in (10) not only of the original essential multiplicity function $\kappa$, but also of two further functions $\kappa^{+}, \kappa^{-}[10, \S 8]$, which may be regarded as the positive part and the negative part of $\kappa$ respectively. If we now change to the function $K$, we can define in a plausible manner two functions $\mathrm{K}^{+}, \mathrm{K}^{-}$, corresponding to $\kappa^{+}, \kappa^{-}$respectively. The functions $\mathrm{K}^{+}, \mathrm{K}^{-}$have properties analogous to those described for $\kappa^{+}, \kappa^{-}$in $(10, \S \S 8-16)$. By methods similar to those in [5] it can be shown that $\mathrm{K}^{+}=\kappa^{+}, \mathrm{K}^{-}=\kappa^{-}$excepting possibly at a countable set of points. In other words, the functions $\kappa, \kappa^{+}, \kappa^{-}$may be replaced by $\mathrm{K}, \mathrm{K}^{+}, \mathrm{K}^{-}$respectively throughout the entire theory for the 2 -cell case. This theory then appears to be formulated in terms of basic concepts which presently appear most suitable for generalization to higher dimensions. The writers plan to extend their work on the transformation of double integrals to the general $n$-dimensional case by utilizing these concepts.

\section{Preliminaries}

2.1. In the sequel, $\Re$ denotes a bounded simply connected Jordan region in Euclidean two-space $E_{2}$, and $\Re^{0}$ indicates the interior of $\Re$. Since $\Re$ is fixed throughout, it will appear in notations only when clarity requires its 
presence. In $\Re^{0}$ we study real, finite-valued functions $f$ of the following type.

(i) At every point $w$ in $\Re^{0}$ where $f$ is defined, its value $f(w)$ is computed by an explicitly given limit process. Conversely, $f$ is defined at every point in $\Re^{0}$ where the limit exists. The exceptional set of those points $w$ in $\Re^{0}$ where the limit fails to exist is denoted by $e(f)$. Thus $f$ is a real, finite-valued function whose range of definition is precisely $\Re^{0}-e(f)$.

(ii) The exceptional set $e(f)$ has (two-dimensional) measure zero.

(iii) The function $f(w)$, we $\Re^{0}-e(f)$, is measurable. It will occasionally happen that two different limit processes yield functions $f_{1}$ and $f_{2}$ of the type described above, such that $f_{1}(w)=f_{2}(w)$ for $w \in \Re^{0}-\left[e\left(f_{1}\right)+e\left(f_{2}\right)\right]$. Then $f_{1}$ and $f_{2}$ differ only on a set of measure zero and are interchangeable for many purposes. But we shall have to distinguish carefully between the case when $f_{1}$ and $f_{2}$ are equal on the common part of their ranges of definition but their ranges of definition differ, and the case when $f_{1}$ and $f_{2}$ are identical-that is, they have exactly the same exceptional sets. For clarity we use the notation $f_{1} \equiv f_{2}$ only when $e\left(f_{1}\right)=e\left(f_{2}\right)$ and $f_{1}(w)=f_{2}(w)$ for $w \in \Re^{0}-e\left(f_{1}\right)=\Re^{0}-e\left(f_{2}\right)$.

2.2. Let $f$ be a function of the type described in 2.1. Then $f$ is said to have an approximate limit $\alpha$ at a point $w_{0}$ in $\Re^{0}$, if for every positive number $\epsilon$, the point $w_{0}$ is a point of density of the set of points $w \in \Re^{0}-e(f)$ where $|f(w)-\alpha| \leqq \epsilon[1$, VII, §3]. The approximate limit $\alpha$ is unique, and is denoted by $f^{*}\left(w_{0}\right)$ in the sequel. Observe that $f^{*}$ may be defined at a point where $f$ is not defined, and conversely. The function $f^{*}$ is regarded as the result of the original limit process which yields $f$ followed by the approximate limit process - that is, the superscript ${ }^{*}$ is thought of as an operator. Since $f$ is approximately continuous almost everywhere in $\Re^{0}[1, \mathrm{IV}, \S 10]$, it is evident that $f^{*}$ is also a function of the type described in 2.1 , and that $f=f^{*}$ almost everywhere in $\Re^{0}$ — the set of points in $\Re^{0}$ where the relation $f=f^{*}$ fails to hold is denoted by $e^{*}(f)$ in the sequel. However, the relation $f \equiv f^{*}$ does not generally hold, and the sets $e(f)$ and $e\left(f^{*}\right)$ show no relationship to each other; of course, $e^{*}(f) \supset e(f)+e\left(f^{*}\right)$.

2.3. The following facts for functions of the type described in 2.1 are easy consequences of the properties of approximate limits [1, VII ].

(i) $f_{1}=f_{2}$ a.e. in $\Re^{0}$ if and only if $f_{1}^{*} \equiv f_{2}^{*}$ (see 2.1).

(ii) Let $e$ be any set in $\Re^{0}$ of measure zero. For any finite set of functions $f_{i}$ for $i=1, \cdots, m$ and any point $w_{0}$ of $\Re^{0}$ not in $\sum_{i=1}^{m} e\left(f_{i}^{*}\right)$ one has a sequence of distinct points $w_{n}$ not in $e+\sum_{i=1}^{m} e\left(f_{i}\right)+\sum_{i=1}^{m} e\left(f_{i}^{*}\right)$ such that $f_{i}\left(w_{n}\right)=f_{i}^{*}\left(w_{n}\right)$ and $f_{i}^{*}\left(w_{n}\right)$ converges to $f_{i}^{*}\left(w_{0}\right)$ for $i=1, \cdots, m$. This fact may be used to prove some of the subsequent statements.

(iii) $f^{* *} \equiv f^{*}$ since $f^{*}=f$ a.e. in $\Re^{0}$ (see (i)).

(iv) If $f_{1} \leqq f_{2}$ a.e. in $\Re^{0}$, then $f_{1}^{*} \leqq f_{2}^{*}$ on $\Re^{0}-\left(e\left(f_{1}^{*}\right)+e\left(f_{2}^{*}\right)\right)$.

(v) $e\left(|f|^{*}\right) \subset e\left(f^{*}\right)$ and $|f|^{*}=\left|f^{*}\right|$ on $\Re^{0}-e\left(f^{*}\right)$.

(vi) If $c_{1}$ and $c_{2}$ are real constants, then $e\left[\left(c_{1} f_{1}+c_{2} f_{2}\right)^{*}\right] \subset e\left(f_{1}^{*}\right)+e\left(f_{2}^{*}\right)$ and $\left(c_{1} f_{1}+c_{2} f_{2}\right)^{*}=c_{1} f_{1}^{*}+c_{2} f_{2}^{*}$ on $\Re^{0}-\left(e\left(f_{1}^{*}\right)+e\left(f_{2}^{*}\right)\right)$. 
(vii) $e\left[\left(f_{1} f_{2}\right)^{*}\right] \subset e\left(f_{1}^{*}\right)+e\left(f_{2}^{*}\right)$ and $\left(f_{1} f_{2}\right)^{*}=f_{1}^{*} f_{2}^{*}$ on $\Re^{0}-\left(e\left(f_{1}^{*}\right)+e\left(f_{2}^{*}\right)\right)$.

(viii) $e\left[\left(\left\{f_{1}^{2}+f_{2}^{2}+f_{3}^{2}\right\}^{1 / 2}\right)^{*}\right] \subset e\left(f_{1}^{*}\right)+e\left(f_{2}^{*}\right)+e\left(f_{3}^{*}\right)$ and $\left(\left\{f_{1}^{2}+f_{2}^{2}+f_{3}^{2}\right\}^{1 / 2}\right)^{*}$ $=\left\{f_{1}^{* 2}+f_{2}^{* 2}+f_{3}^{* 2}\right\}^{1 / 2}$ on $\Re^{0}-\left(e\left(f_{1}^{*}\right)+e\left(f_{2}^{*}\right)+e\left(f_{3}^{*}\right)\right)$.

2.4. In the sequel we have to study situations in which there is associated with each direction $o x$ in Euclidean three-space $E_{3}$ a function $f_{x}$ of the type described in 2.1. A right-handed system of coordinates $o x^{1} x^{2} x^{3}$ constitutes a frame of reference $X$ in $E_{3}$. Let $f_{X}(w)$ denote the vector with components $f_{x^{1}}(w), f_{x^{2}}(w), f_{x^{3}}(w)$ defined at each point $w$ of $\Re^{0}$ not in the set $\sum_{i=1}^{3} e\left(f_{x^{i}}\right)$. This latter set is of measure zero, and is denoted by $e\left(f_{X}\right)$ in the sequel. At a point of $\Re^{0}$ not in $e\left(f_{X}\right),\left|f_{X}\right|$ is the length of the vector $f_{X}$. Analogously, the symbol $f_{X}^{*}$ is defined at each point of $\Re^{0}$ not in the set $e\left(f_{X}^{*}\right)=\sum_{i=1}^{3} e\left(f_{x^{i}}^{*}\right)$ to be the vector with components $f_{x^{1}}^{*}, f_{x^{2}}^{*}, f_{x^{3}}^{*}$.

2.5. Lemma. Assume that with each direction ox in $E_{3}$ there is associated a function $f_{x}$ of the type described in 2.1. Suppose that for each direction ox and each frame of reference $X$ there is a set $e(x, X)$ in $\Re^{0}$ of measure zero and depending upon both $x$ and $X$ such that

$$
f_{x}=f_{x i} \cos x^{i} o x \text { on } \Re^{0}-e(x, X),
$$

where the repeated index $i$ indicates a summation over the range of $i$. Then the set $e\left(f_{X}^{*}\right)$ is independent of $X$ and is denoted simply by $e^{*}$. For any direction oy and any frame of reference $Y$,

$$
f_{y}^{*}=f_{y^{i}}^{*} \cos y^{j} \text { oy on } \Re^{0}-e^{*} .
$$

Finally, for any two frames of reference $X$ and $Y$,

$$
\left|f_{Y}^{*}\right|=\left|f_{X}^{*}\right| \text { on } \Re^{0}-e^{*} \text {. }
$$

Proof. From 2.3 (i), (vi) and relation (1) it follows for any direction $o x$ and any frame of reference $X$,

$$
\begin{gathered}
e\left(f_{x}^{*}\right) \subset e\left(f_{X}^{*}\right), \\
f_{x}^{*}=f_{x^{i}}^{*} \cos x^{i} o x \text { on } \Re^{0}-e\left(f_{X}^{*}\right) .
\end{gathered}
$$

In particular, from (4) one obtains for a frame of reference $Y$ the relation $e\left(f_{Y}^{*}\right)=\sum_{i=1}^{3} e\left(f_{\nu^{i}}^{*}\right) \subset e\left(f_{X}^{*}\right)$, and in view of the symmetry, the fact that $e\left(f_{X}^{*}\right)$ does not depend upon $X$ is established. From (5) one obtains $f_{y^{i}}^{*}=f_{x^{i}}^{*} \cos x^{i} o y^{j}$ on $\mathfrak{R}^{0}-e^{*}$. Multiplying both sides by $\cos y^{j} o x^{k}$ and summing yields $f_{y^{i}}^{*} \cos y^{i} o x^{k}=f_{x^{*}}^{*}$ on $\Re^{0}-e^{*}$. Then from relation (5)

$$
f_{y}^{*}=f_{x^{i}}^{*} \cos x^{i} o y=f_{y^{i}}^{*} \cos y^{j} o x^{i} \cos x^{i} o y=f_{y^{i}}^{*} \cos y^{j} o y \text { on } \Re^{0}-e^{*},
$$

and relation (2) is established. Relation (3) follows immediately from (2).

2.6. Lemma. Assume that with each direction ox in $E_{3}$ there is associated a 
function $f_{x}$ satisfying all the hypotheses of the lemma in 2.5. Suppose that $h$ is a function of the type described in 2.1 which has the following property: For each frame of reference $X$ there is a set $e(X)$ in $\Re^{0}$ of measure zero and depending upon $X$ such that

$$
\left|f_{X}\right| \leqq h \leqq \sum_{i=1}^{3}\left|f_{x^{i}}\right| \text { on } \Re^{0}-e(X) \text {. }
$$

$P u t e^{* *}=e\left(h^{*}\right)+e^{*}, e^{*}=e^{*}(h)+e^{* *}$ where $e^{*}$ is the set described in 2.5 (see $\left.\$ 2.2\right)$. Then $e^{* *}$ and $e^{\star}$ are sets of measure zero in $\Re^{0}$, and for any frame of reference $Y$,

$$
\begin{gathered}
h^{*}=\left|f_{Y}^{*}\right| \text { on } \Re^{0}-e^{* *}, \\
h=\left|f_{Y}^{*}\right| \text { on } \Re^{0}-e^{*} .
\end{gathered}
$$

Consequently

$$
h=\left|f_{Y}\right| \text { a.e. on } \Re^{0} \text {, }
$$

where the exceptional set in relation (4) depends upon the frame $Y$.

Proof. From relation (1) and 2.3 (iv), (v), (vi), (viii) one finds that for every frame $X$,

$$
\left|f_{X}^{*}\right| \leqq h^{*} \leqq \sum_{i=1}^{3}\left|f_{x^{i}}^{*}\right| \text { on } \Re^{0}-e^{* *},
$$

and from 2.5 (3) one has

$$
\left|f_{X}^{*}\right|=\left|f_{Y}^{*}\right| \text { on } \Re^{0}-e^{* *},
$$

for any two frames $X$ and $Y$.

Case (i). Suppose that at a particular point of $\Re^{0}-e^{* *},\left|f_{Y}^{*}\right|=0$. From (5) and (6) it is evident that $h^{*}=0$, and thus (2) is established in this case.

Case (ii). Suppose that at a particular point of $\Re^{0}-e^{* *},\left|f_{Y}^{*}\right|>0$. Choose a frame of reference $X$ so that at this point, one has

$$
f_{x^{1}}^{*}=\left|f_{Y}^{*}\right|, \quad f_{x^{2}}^{*}=o=f_{x^{3}}^{*} .
$$

From relations (5) and (6) it is clear that (2) must be valid in this case also. Relations (3) and (4) follow at once from 2.2.

2.7. When the hypotheses are suitably strengthened, the operator * may be dropped in the results contained in 2.5 and 2.6. By the methods used in the proofs of these results, the reader will easily establish the following facts.

LEMma. Assume that with each direction ox in $E_{3}$ there is associated a func- 
tion $f_{x}$ of the type described in 2.1. Suppose that for a certain frame of reference $X$ there is a set $e^{\prime}$ in $\Re^{0}$ of measure zero and such that for every direction ox

$$
e^{\prime} \supset e\left(f_{x}\right) \text { and } f_{x}=f_{x^{i}} \cos x^{i} o x \text { on } \Re^{0}-e^{\prime} .
$$

Then for any direction oy and any frame of reference $Y$,

$$
f_{y}=f_{y^{i}} \cos y^{i} o y \text { on } \Re^{0}-e^{\prime}
$$

and

$$
\left|f_{Y}\right|=\left|f_{X}\right| \text { on } \Re^{0}-e^{\prime} \text {. }
$$

Moreover, if $h$ is a function of the type described in 2.1 for which there is a set $e^{\prime \prime}$ in $\Re^{0}$ of measure zero and containing $e^{\prime}$ such that for any frame $Y$,

$$
\left|f_{Y}\right| \leqq h \leqq \sum_{i=1}^{3}\left|f_{y^{i}}\right| \text { on } \Re^{0}-e^{\prime \prime}
$$

then

$$
h=\left|f_{Y}\right| \text { on } \Re^{0}-e^{\prime \prime} .
$$

2.8. The following result, which the reader may readily verify, contains a sufficient condition in order that the hypotheses of the lemma in 2.7 be fulfilled. In the sequel we assume that a rectangular Cartesian coordinate system is fixed in the $w$-plane, with reference to which we speak of oriented rectangles $R$ in $\Re^{0}$.

LEMмA. Assume that with each direction ox in $E_{3}$ there is associated a func-

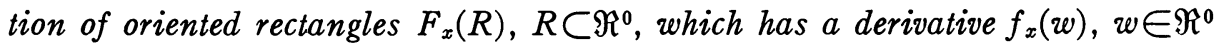
$-e\left(f_{x}\right)$, of the type described in 2.1. Suppose that for a certain frame of reference $X$ and for every direction ox,

$$
F_{x}(R)=F_{x^{i}}(R) \cos x^{i} o x \text { for } R \subset \Re^{0} .
$$

Then $f_{x}$ satisfies condition 2.7 (1) with $e^{\prime}=e\left(f_{X}\right)$ (see 2.4). Moreover, if $H(R)$, $R \subset \Re^{0}$, is a function of oriented rectangles having a derivative $h(w), w \in \Re^{0}$ $-e(h)$, of the type described in 2.1 such that for every frame $Y$,

$$
\left|F_{Y}(R)\right| \leqq H(R) \leqq \sum_{i=1}^{3}\left|F_{y^{i}}(R)\right| \text { for } R \subset \Re^{0}
$$

where $F_{Y}(R)$ is the vector with components $F_{y^{1}}(R), F_{y^{2}}(R), F_{y^{3}}(R)$, then condition 2.7 (4) is satisfied by the derivatives $f_{Y}$ and $h$ when $e^{\prime \prime}$ is chosen to be $e^{\prime}+e(h)$.

2.9. Lemma. Assume that with each direction ox in $E_{3}$ there is associated a function of oriented rectangles $F_{x}(R), R \subset \Re^{0}$, which has a derivative $f_{x}(w)$, $w \in \Re^{0}-e\left(f_{x}\right)$, of the type described in 2.1. Suppose $G(R), R \subset \Re^{0}$, is a function of oriented rectangles having a derivative $g(w), w \in \Re^{0}-e(g)$, of the type described 
in 2.1 such that for any pair of directions ox and oy,

$$
\left|F_{x}(R)-F_{y}(R)\right| \leqq \rho(x \circ y) G(R) \text { for } R \subset \Re^{0},
$$

where $\rho(x o y)$ is a function of the angle xoy which converges to zero with that angle. Then there is a set $e^{\prime \prime \prime}$ in $\Re^{0}$ of measure zero such that for any direction ox,

$$
e^{\prime \prime \prime} \supset e\left(f_{x}\right),
$$

and for any pair of directions ox and oy,

$$
\left|f_{x}(w)-f_{y}(w)\right| \leqq \rho(x o y) g(w) \quad \text { for } \quad w \in \Re^{0}-e^{\prime \prime \prime} .
$$

Proof. Choose a dense countable set 8 of directions $o z$ in $E_{3}$, and put $e^{\prime \prime \prime}=e(g)+\sum e\left(f_{z}\right)$ for $o z$ in 3 . Evidently $e^{\prime \prime \prime}$ is a set of measure zero in $\Re^{0}$. Let $w$ be any point in $\Re^{0}-e^{\prime \prime \prime}$, and $o x$ any direction in $E_{3}$. Denote by $\Delta$ and $\delta$ the upper and lower derivates respectively of $F_{x}$ at $w$. From (1), it follows that for any direction $o z$ in $3,\left|\Delta-f_{z}(w)\right| \leqq \rho(x o z) g(w)$ and $\left|\delta-f_{z}(w)\right|$ $\leqq \rho(x o z) g(w)$, hence $|\Delta-\delta| \leqq 2 \rho(x o z) g(w)$. Since the directions oz in $\mathbb{B}$ are dense, and $\rho(x o z)$ tends to zero with $x o z$, it follows that $\Delta=\delta=f_{x}(w)$. Thus relation (2) is established, and relation (3) follows at once from (1), (2) and the fact that $e^{\prime \prime \prime}$ contains $e(g)$.

2.10. Lemma. Assume that with each direction ox in $E_{3}$ there are associated functions of oriented rectangles $F_{i x}(R), R \subset \Re^{0}, i=1,2$, which have derivatives $f_{i x}(w)$, we $\Re^{0}-e\left(f_{i x}\right)$, of the type described in 2.1. Suppose $G_{i}(R), R \subset \Re^{0}$, $i=1,2$, are functions of oriented rectangles having derivatives $g_{i}(w), w \in \Re^{0}$ $-e\left(g_{i}\right)$, of the type described in 2.1 such that for any pair of directions ox and oy,

$$
\left|F_{i x}(R)-F_{i y}(R)\right| \leqq \rho_{i}(x \circ y) G_{i}(R) \quad \text { for } \quad R \subset \Re^{0}, \quad i=1,2,
$$

where the $\rho_{i}(x \circ y)$ for $i=1,2$ are functions of the angle xoy which converge to zero with that angle. Finally, assume that for each direction ox there is a set $e(x)$ in $\Re^{0}$ of measure zero such that $f_{1 x}=f_{2 x}$ on $\Re^{0}-e(x)$. Then there is a set $e^{\prime \prime \prime \prime}$ in $\Re^{0}$ of measure zero such that for any direction ox,

$$
e^{\prime \prime \prime \prime} \supset e\left(f_{i x}\right)
$$$$
\text { for } i=1,2
$$

and

$$
f_{1 x}=f_{2 x} \text { on } \Re^{0}-e^{\prime \prime \prime \prime} .
$$

Proof. Let $e_{i}^{\prime \prime \prime}$ for $i=1,2$, be the set described in 2.9 which corresponds to the functions $F_{i x}$ and $G_{i}$ for $i=1,2$. Choose a dense countable set 3 of directions $o z$ in $E_{3}$ and put $e^{\prime \prime \prime \prime}=e_{1}^{\prime \prime \prime}+e_{2}^{\prime \prime \prime}+\sum e(z)$ for $o z$ in 3 . Evidently $e^{\prime \prime \prime \prime}$ is a set of measure zero in $\Re^{0}$, and relation (2) holds. Let $w$ be a point in $\Re^{0}-e^{\prime \prime \prime \prime}$, and $o x$ any direction in $E_{3}$. From 2.9(3) and the assumptions, it follows for any direction $o z$ in 3 that

$$
\left|f_{1 x}(w)-f_{2 x}(w)\right| \leqq \rho_{1}(x o z) g_{1}(w)+\rho_{2}(x o z) g_{2}(w) .
$$




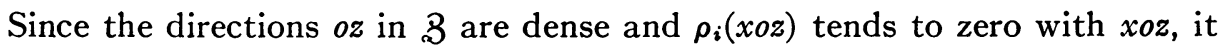
follows that $f_{1 x}(w)=f_{2 x}(w)$, and (3) is established.

\section{ApPLICATIONS}

3.1. Again $\Re$ is a bounded simply connected Jordan region in $E_{2}$ (see $\$ 2.1$ ) and a rectangular Cartesian coordinate system is fixed in the plane of $\Re$ as in $\S 2.8$. Let $T: \Re \rightarrow E_{3}$ be a continuous mapping from $\Re$ into Euclidean threespace $E_{3}$. Since $T$ and $\Re$ are kept fixed in this paper, they are displayed in subsequent notations only when clarity requires it. Now $T: \Re \rightarrow E_{3}$ represents an $F$-surface of the type of the 2-cell [6; II.3.44]. It is assumed in the sequel that this surface has finite Lebesgue area or, equivalently, that $T$ is an eBV representation $[6 ;$ V.1.15, 2.61, 2.65]. Presently some well known facts are listed to which the results in the preceding chapter are applied.

3.2 . Let $R$ be an oriented rectangle in $\Re$ (see $\$ 2.8$ ). Then $T: R \rightarrow E_{3}$ determines a $F$-surface whose Lebesgue area is denoted by $A(R)$ for $R$ in $\Re$. The function of oriented rectangles $A(R), R \subset \Re$, possesses a derivative $D(w, A)$ almost everywhere in $\Re^{0}$ which is summable in $\Re^{0}[6 ; \mathrm{V} .2 .18]$, and hence of the type described in $\$ 2.1$. The exceptional set $e(D(w, A))$ is denoted by $e^{1}$. It is important to observe that the function $A(R), R \subset \Re$, and hence its derivative $D(w, A), w \in \Re^{0}-e^{1}$, are independent of the choice of a frame of reference in $E_{3}[6 ;$ V.2.8].

3.3. Let $o x$ be any direction in $E_{3}$. Then $T_{x}: \Re \rightarrow E_{2}$ will denote the continuous plane transformation obtained by going from $\Re$ into $E_{3}$ under $T$ and then projecting orthogonally into the plane $\pi_{x}$ through $o$ with normal $o x$. Since $T$ is eBV by assumption, it follows that $T_{x}$ is eBV in $\Re^{0}[6 ; \mathrm{V} .1 .15]$. Consequently $T_{x}$ gives rise to a number of important functions which depend essentially upon the direction $o x$. A description of the ones needed in the sequel follows. Close inspection of their definitions shows that they are independent of the choice of a frame of reference in $\pi_{x}$. The symbol $\mathfrak{x}$ stands for a generic point in the plane $\pi_{x}$.

3.4. Let $R$ be an oriented rectangle in $\Re$, and $o x$ any direction in $E_{3}$. Then the mapping $T_{x}: R^{0} \rightarrow E_{2}$ gives rise to the essential multiplicity $\kappa\left(\mathfrak{x}, x, R^{0}\right)$ of each point $\mathfrak{x}$ in the plane $\pi_{x}$ in the image of $R^{0}$ under $T_{x}[6 ;$ IV.1.43, 1.47]. Since $T_{x}$ is eBV in $R^{0}\left[6 ;\right.$ IV.4.1], $\kappa\left(\mathfrak{x}, x, R^{0}\right)$ is summable in $\pi_{x}$; denote its integral over $\pi_{x}$ by $G(R, x, \kappa)$-this is the function $G_{4}^{*}$ of $[6 ;$ IV.3.8]. Now $G(R, x, \kappa), R \subset \Re$, has a derivative $D(w, x, \kappa)$ almost everywhere in $\Re^{0}$ which is summable in $\Re^{0}[6 ;$ IV.3.13]), and hence of the type described in $\S 2.1$. The exceptional set $e(D(w, x, \kappa))$ is denoted by $e^{2}(x)$ in the sequel. For a frame of reference $X$ in $E_{3}$ given by coordinates $o x^{1} x^{2} x^{3}$, the set $\sum_{i=1}^{3} e^{2}\left(x^{i}\right)$ is denoted by $e^{2}(X)$. At each point $w$ of $\Re^{0}$ not in $e^{2}(X), D(w, X, \kappa)$ is the vector with components $D\left(w, x^{1}, \kappa\right), D\left(w, x^{2}, \kappa\right), D\left(w, x^{3}, \kappa\right)$.

3.5. Retain the assumptions made in $\S 3.4$. Let $g(R, x, \kappa)$ for $R \subset \Re$ be the integral over the plane $\pi_{x}$ of the characteristic function $c\left(x, x, R^{0}\right)$ of the set 
of points in $\pi_{x}$ where $\kappa\left(x, x, R^{0}\right)$ is not zero-this is the function $G_{4}$ of [6; IV.3.7]. Now $g(R, x, \kappa), R \subset \Re$, has a derivative $d(w, x, \kappa)$ almost everywhere in $\Re^{0}$ which is summable in $\Re^{0}$ [6; IV.3.13], and hence of the type described in $\$ 2.1$. The exceptional set $e(d(w, x, \kappa))$ is denoted by $e^{3}(x)$ in the sequel. Moreover [6; IV.3.13] $d(w, x, \kappa)=D(w, x, \kappa)$ almost everywhere in $\Re^{0}$.

3.6. Retain the working hypotheses of $\$ 3.5$. At each point $w$ of $\Re^{0}-e^{3}(x)$ the essential generalized Jacobian $\jmath_{e}(w, x)$ is defined [6, IV.3.21]. Evidently $J_{e}(w, x), w \in \Re^{0}-e^{3}(x)$, is again a function of the type described in $\$ 2.1$. For a frame of reference $X$ in $E_{3}$ given by coordinates $o x^{1} x^{2} x^{3}$, the set $\sum_{i=1}^{3} e^{3}\left(x^{i}\right)$ is denoted by $e^{3}(X)$ (see $\S 2.4$ ). At each point $w$ of $\Re^{0}$ not in $e^{3}(X), J_{e}(w, X)$ is the vector with components $J_{e}\left(w, x^{1}\right), J_{e}\left(w, x^{2}\right), J_{e}\left(w, x^{3}\right)$.

3.7. Continue with the assumptions of $\$ 3.4$. The mapping $T_{x}: R^{0} \rightarrow E_{2}$ gives rise to non-negative multiplicity functions $\kappa^{+}\left(\mathfrak{x}, x, R^{0}\right), \kappa^{-}\left(\mathfrak{x}, x, R^{0}\right)$ whose sum is $\kappa\left(\mathfrak{x}, x, R^{0}\right)$ for $R \subset \Re[10 ; \S 8]$. Both $\kappa^{+}$and $\kappa^{-}$are summable in $\pi_{x}$; let $G(R, x)$ denote the integral of $\kappa^{+}\left(\mathfrak{x}, x, R^{0}\right)-\kappa^{-}\left(\mathfrak{x}, x, R^{0}\right)$ over $\pi_{x}$-this is the function $G^{\odot}$ of $[10 ; \S 23]$. Then $G(R, x)$ has a derivative $D(w, x)$ almost everywhere in $\Re^{0}$ which is summable in $\Re^{0}[10 ; \S 23]$, and hence of the type described in $\$ 2.1$. The exceptional set $e(D(w, x))$ is denoted by $e^{4}(x)$ in the sequel. For a frame of reference $X$ in $E_{3}, e^{4}(X)$ is the set $\sum_{i=1}^{3} e^{4}\left(x^{i}\right)$, and at each point $w$ of $\Re^{0}$ not in $e^{4}(X), D(w, X)$ is the vector with components $D\left(w, x^{1}\right), D\left(w, x^{2}\right), D\left(w, x^{3}\right)$.

3.8. Recall the conditions set forth in $\$ 3.3$. If the mapping $T_{x}: \Re \rightarrow E_{2}$ possesses an ordinary Jacobian $J(w, x)$ at a point $w$ in $\Re^{0}[6, I V .3 .21]$, it is well known that neither its value nor its existence is dependent upon the choice of a frame of reference in $\pi_{x}$. Let $E(x)$ denote the set of points in $\Re^{0}$ where $T_{x}$ fails to possess an ordinary Jacobian. Then $E(x)$ is not generally of measure zero when $T_{x}$ is eBV in $\Re^{0}$-in fact, $E(x)$ may be identical with $\Re^{0}$. Thus the function $J(w, x), w \in \Re^{0}-E(x)$, is not generally of the type described in $\$ 2.1$. For a frame of reference $X$ in $E_{3}$, let $E(X)$ be the set $\sum_{i=1}^{3} E\left(x^{i}\right)$ and at each point $w$ of $\Re^{0}$ not in $E(X)$, let $J(w, X)$ be the vector with components $J\left(w, x^{1}\right), J\left(w, x^{2}\right), J\left(w, x^{3}\right)$. It is well known that $E(X)$ does not depend upon the vector $X$, hence it is denoted simply by $E^{5}$. For any direction $o y$, any frame of reference $Y$, one has

$$
J(w, y)=J\left(w, y^{j}\right) \cos y^{j} o y \text { on } \Re^{0}-E^{5} .
$$

3.9. Given a frame of reference $X$ and a direction $o x$ in $E_{3}$, the function of rectangles $G(R, x), R \subset \Re$, introduced in $\$ 3.7$ satisfies the relation $G(R, x)$ $=G\left(R, x^{i}\right) \cos x^{i} o x$ for $R \subset \Re[10 ; \S \S 5,29]$. From the lemmas in $\$ 2.8$ and $\S 2.7$ follows the existence of a set $e^{6} \equiv e^{4}(X)$ in $\Re^{0}$ of measure zero and such that for any direction $o y$ and any frame of reference $Y, e^{6} \supset e^{4}(y)$ and

$$
\begin{aligned}
D(w, y) & =D\left(w, y^{j}\right) \cos y^{j} o y \text { on } \Re^{0}-e_{.}^{6}, \\
|D(w, Y)| & =|D(w, X)| \text { on } \Re^{0}-e^{6} .
\end{aligned}
$$


3.10. For any direction $o x$ it is known that $\Im_{e}(w, x)=D(w, x)$ almost everywhere in $R^{0}[10 ; \S \S 5,23]$. In view of $\$ 3.9$ (1) it follows that for any frame of reference $X$ in $E_{3}$, one has $J_{e}(u, x)=J_{e}\left(u, x^{i}\right) \cos x^{i} o x$ almost everywhere in $\Re^{0}$, where the exceptional set now depends upon both $x$ and $X$. From the lemma in $\$ 2.5$ follows the existence of a set $e^{7}$ in $\Re^{0}$ of measure zero and such that for any direction oy and any frame of reference $Y$, $e^{7} \supset e\left(J_{e}^{*}(w, y)\right)+e^{6}$ and

$$
\begin{aligned}
J_{e}^{*}(w, y) & =J_{e}^{*}\left(w, y^{j}\right) \cos y^{j} o y \text { on } \Re^{0}-e^{7}, \\
\left|J_{e}^{*}(w, Y)\right| & =\left|J_{e}^{*}(w, X)\right| \text { on } \Re^{0}-e^{7}, \\
J_{e}^{*}(w, y) & =D(w, y) \text { on } \Re^{0}-e^{7} .
\end{aligned}
$$

3.11. For any direction $o x$ it is known that $|D(w, x)|=D(w, x, \kappa)$ almost everywhere in $\Re^{0}[6 ;$ IV.3.25, 3.13], where the exceptional set depends upon the direction $0 x$. However, it will now be shown that there is a set $e^{8}$ in $\Re^{0}$ of measure zero and such that for any direction $o y, e^{8} \supset e^{2}(y)+e^{4}(y)$ and

$$
|D(w, y)|=D(w, y, \kappa) \text { on } \Re^{0}-e^{8} \text {. }
$$

In view of the lemma in $\$ 2.10$, this fact is established by the result in the next section.

3.12. Lemma. The functions of oriented rectangles $A(R), G(R, x, \kappa), G(R, x)$, $R \subset \Re$, introduced in $\$ \S 3.2,3.4,3.7$ respectively, satisfy the following conditions. For any pair of directions ox and oy,

$$
\begin{aligned}
& |G(R, x, \kappa)-G(R, y, \kappa)| \leqq 2 \sin x o y / 2 \cdot A(R) \text { for } R \subset \Re, \\
& |G(R, x)-G(R, y)| \leqq 2 \sin x o y / 2 \cdot A(R) \text { for } R \subset \Re .
\end{aligned}
$$

Proof. Case (i). Assume that the mapping $T: \Re \rightarrow E_{3}$ is eAC [6; V.1.15]; it is known that this property is independent of the choice of the frame of reference in $E_{3}[10 ; \S 30]$. Then for any direction $o x$ in $E_{3}, T_{x}$ is eAC in $\Re^{0}$, and consequently $[6 ;$ IV.4.7; $10 ; \S 24]$

$$
\begin{aligned}
G(R, x, \kappa) & =\iint_{R} D(w, x, \kappa)=\iint_{R}|D(w, x)|, \\
G(R, x) & =\iint_{R} D(w, x) .
\end{aligned}
$$

For any frame of reference $X$, it follows from $\$ 3.10$ (1) and $\$ 3.11$ that

$$
|D(w, X)|=\left|J_{e}(w, X)\right|
$$

almost everywhere in $\Re^{0}$, and consequently [6; V.2.61] 


$$
A(R)=\iint_{R}|D(w, X)|
$$

Thus the inequalities in (1) are established if one proves that

$$
\iint_{R}|D(w, x)-D(w, y)| \leqq 2 \sin x o y / 2 \cdot \iint_{R}|D(w, X)| .
$$

But this inequality follows immediately from $\$ 3.9$ (1) and the inequality of Schwarz.

Case (ii). Assume that $T$ is merely eBV (see $\$ 3.1$ ). Then there is a sequence of continuous mappings $T_{n}: R \rightarrow E_{3}$ each of which is eAC-in fact, each may be chosen to represent a polyhedron $[6 ; \mathrm{V} .2 .5]$-and such that the $T_{n}$ converge uniformly to $T$ on $R$ and their Lebesgue areas $A_{n}(R)$ converge to $A(R)$. Let $G_{n}(R, x, \kappa)$ and $G_{n}(R, x)$ be the functions of oriented rectangles generated by $T_{n}$ which correspond to $G(R, x, \kappa)$ and $G(R, x)$ respectively for $T$ (see $\S \S 3.4,3.7)$. From case (i); $[9, \S 3] ;[10, \S 26]$ one obtains the following relations:

$$
\begin{array}{cc}
\left|G_{n}(R, x, \kappa)-G_{n}(R, y, \kappa)\right| \leqq 2 \sin x o y / 2 \cdot A_{n}(R) & \text { for } n=1,2, \cdots, \\
\left|G_{n}(R, x)-G_{n}(R, y)\right| \leqq 2 \sin x o y / 2 \cdot A_{n}(R) & \text { for } n=1,2, \cdots, \\
\lim _{n \rightarrow \infty} G_{n}(R, x, \kappa)=G(R, x, \kappa), & \lim _{n \rightarrow \infty} G_{n}(R, y, \kappa)=G(R, y, \kappa), \\
\lim _{n \rightarrow \infty} G_{n}(R, x)=G(R, x), & \lim _{n \rightarrow \infty} G_{n}(R, y)=G(R, y) .
\end{array}
$$

Clearly the inequalities in (1) follow.

3.13. Given $T$ as in $\$ 3.1$, the functions of rectangles $A(R), G(R, x, \kappa)$, $R \subset \Re$, introduced in $\$ \S 3.2,3.4$ respectively satisfy the following condition $[6 ;$ V.1.2, 1.3, 1.16, 2.60, 2.65]. For every frame of reference $Y$,

$$
|G(R, Y, \kappa)| \leqq A(R) \leqq \sum_{i=1}^{3}\left|G\left(R, y^{i}, \kappa\right)\right| \text { for } \quad R \subset \Re .
$$

In view of $\$ 3.11(1)$, it follows from this relation that

$$
|D(w, Y)| \leqq D(w, A) \leqq \sum_{i=1}^{3}\left|D\left(w, y^{i}\right)\right| \text { on } \Re^{0}-\left(e^{1}+e^{8}\right) .
$$

From $\$ 3.9$ (1) and $\$ 3.11$ (1) follows by the lemma in $\$ 2.7$ the existence of a set $e^{9}$ in $\Re^{0}$ of measure zero and such that $e^{9} \supset e^{1}+e^{6}+e^{8}$ and for every frame of reference $Y$,

$$
D(w, A)=|D(w, Y)| \text { on } \Re^{0}-e^{9} .
$$

3.14. The information derived in the preceding sections on relations which hold almost everywhere uniformly with respect to the frame of reference is 
summarized in the following statement.

THEOREM. Given a continuous mapping $T: \Re \rightarrow E_{3}$ which is eBV (see §3.1), there exists a set $e(T)$ in $\Re^{0}$ of measure zero such that the following statements hold at each point $w$ in $\Re^{0}-e(T)$ for every direction oy and every frame of reference $Y$.

(i) The functions $D(w, A), D(w, y, \kappa), D(w, y), J_{e}^{*}(w, y)$ exist;

(ii) $D(w, y)=\Im_{e}^{*}(w, y)$;

(iii) $D(w, y, \kappa)=|D(w, y)|=\left|J_{e}^{*}(w, y)\right|$;

(iv) $D(w, A)=|D(w, Y)|=\left|\jmath_{e}^{*}(w, Y)\right|=|D(w, Y, \kappa)|$;

(v) $D(w, y)=D\left(w, y^{j}\right) \cos y^{j} o y$;

(vi) $J_{e}^{*}(w, y)=J_{e}^{*}\left(w, y^{i}\right) \cos y^{i} o y$.

A proof follows at once from $\$ \$ 3.9-3.13$ with $e(T)=e^{7}+e^{9}$.

3.15. It will be noticed that the operator $*$ plays a role in this theorem only on the essential generalized Jacobians $J_{e}(w, y)$. The question as to whether this * may be removed seems to involve certain topological problems which we have not solved thus far. However, for a fixed frame of reference $X$, we have (see $\$ 2.2) D(w, A)=\mathfrak{J}_{\epsilon}(w, X)$ almost everywhere in $\Re^{0}$, where the exceptional set now depends upon the frame $X$. This result answers a question raised in $[6 ; V .2 .18]$-namely, that the derivative of the Lebesgue area function $A(R), R \subset \Re$, of a surface having finite area exists almost everywhere in $\Re^{0}$ and equals almost everywhere the square root of the sum of the squares of the three essential generalized Jacobians associated with a given frame of reference.

\section{A THEOREM ON ORDINARY JACOBIANS}

4.1. In the theorem of $\$ 3.14$, no reference to the ordinary Jacobians appears, since these may not exist at a single point. It is interesting to observe that if one adds to the assumptions made in $\$ 3.14$ the hypothesis that the set $E^{5}$ of $\S 3.8$ is of measure zero, one obtains the following result.

Theorem. Given a continuous mapping $T: \Re \rightarrow E_{3}$ which is eBV (see $\$ 3.1$ ), assume that the set $E^{5}$ described in $\$ 3.8$ is of measure zero. There exists a set $E(T)$ in $\Re^{0}$ of measure zero such that $E(T) \supset e(T)$ and the following statements hold at each point $w$ in $\Re^{0}-E(T)$ for every direction oy and every frame of reference $Y$.

(i) The functions $D(w, A), D(w, y), J_{e}^{*}(w, y), J(w, y)$ exist;

(ii) $D(w, y)=\Im_{e}^{*}(w, y)=J(w, y)$;

(iii) $D(w, A)=|D(w, Y)|=\left|\Im_{e}^{*}(w, Y)\right|=|J(w, Y)|$.

Proof. In view of relations $\$ 3.8$ (1) and $\$ 3.9$ (1) it is evident from the theorem in $\$ 3.13$ that this theorem is established if one shows that for any direction $o x$,

$$
D(w, x)=J(w, x) \text { a.e. in } \Re^{0} .
$$


Now the set $E^{5}$ of points $w$ in $\Re^{0}$ where $J(w, x)$ fails to exist is of measure zero by assumption. Put $E^{4}=e^{4}(x)+e^{3}(x)+e^{2}(x)$ (see $\left.\$ \$ 3.4-3.7\right)$; then $E^{4}$ is a set in $\Re^{0}$ of measure zero. Let $E^{3}$ be the set of points $w$ in $\Re^{0}$ not in $E^{4}$ where $d(w, x, \kappa) \neq D(w, x, \kappa)$; then $E^{3}$ is a set of measure zero (see $\S 3.5$ ). A set $E^{2}$ in $\Re^{0}$ is defined by saying that a point $w_{0}$ not in $E^{5}$ is not in $E^{2}$ if the following conditions are fulfilled. Suppose the transformation $T_{x}: \Re \rightarrow E_{2}$ is given by the relation:

$$
T_{x}: \quad x^{k}=x^{k}(w)=x^{k}(u, v), \quad(u, v)=w \in \Re, \quad k=1,2 .
$$

Associate with $w_{0}=\left(u_{0}, v_{0}\right)$ the affine transformation

$$
T_{*}: \quad x^{k}=x^{k}\left(w_{0}\right)+x_{u}^{k}\left(w_{0}\right)\left(u-u_{0}\right)+x_{v}^{k}\left(w_{0}\right)\left(v-v_{0}\right), \quad k=1,2 .
$$

Then there is a sequence of oriented squares $s_{n}$ with center $w_{0}$ whose side lengths converge to zero and for which the constants $m\left(s_{n}\right)$ associated with $T_{*}\left[6 ;\right.$ IV. 3.31] converge to zero as $n$ tends to infinity. The set $E^{2}$ is of measure zero [6; I.3.14; IV.3.42]. Put $E^{1}=E^{2}+E^{3}+E^{4}+E^{5}$; then $E^{1}$ is a set of measure zero in $\Re^{0}$. For $w_{0}$ not in $E^{1}$ it is presently shown that $D\left(w_{0}, x\right)$ $=J\left(w_{0}, x\right)$. It is convenient to consider two cases.

Case (i). Assume that $J\left(w_{0}, x\right)$ is not zero. Since $w_{0}$ is not in $E^{2}$ there follows the existence of a sequence of oriented squares $s_{n}$ in $\Re^{0}$ with center $w_{0}$, with side lengths $l_{n}$ converging to zero, and with values $m\left(s_{n}\right)$ less than $k / 2$ and converging to zero as $n$ tends to infinity [6;IV.3.28, 3.31]. Let $s_{n}^{\prime}, s_{n}^{\prime \prime}$ be oriented squares with center $w_{0}$ and side lengths $l_{n}^{\prime}, l_{n}^{\prime \prime}$ respectively given by

$$
l_{n}^{\prime}=\left(1-2 m\left(s_{n}\right) / k\right) l_{n}, \quad l_{n}^{\prime \prime}=\left(1+2 m\left(s_{n}\right) / k\right) l_{n} .
$$

Let $p_{n}^{\prime}, p_{n}, p_{n}^{\prime \prime}$ denote the parallelograms $T_{*}\left(s_{n}^{\prime}\right), T_{*}\left(s_{n}\right), T_{*}\left(s_{n}^{\prime \prime}\right)$ respectively, and let $q_{n}$ be the doubly connected region composed of points in $p_{n}^{\prime \prime}$ but not in $p_{n}^{\prime}$. From [6; IV 3.35] it follows that

$$
\mu\left(\mathfrak{x}, s_{n}\right)= \begin{cases}0 & \text { for } \mathfrak{x} \operatorname{not} \text { in } p_{n}^{\prime \prime} ; \\ \operatorname{sgn} J\left(w_{0}, x\right) & \text { for } \mathfrak{x} \text { in } p_{n}^{\prime} .\end{cases}
$$

By [6; IV.3.38] it is true that the image of the boundary of $s_{n}$ under $T_{x}$ is contained in the interior of $q_{n}$. Hence from $[2 ; \S 2.20]$ one sees that for almost all points $\mathfrak{x}$ not in $q_{n}, \mu\left(\mathfrak{x}, s_{n}\right)$ is equal to the sum of the generalized local indices of the essential maximal model continua for $x$ under $T_{x}$ in $s_{n}^{0}$. But from $\left[5 ;\right.$ I.1.11, 1.4.6] it is clear that, excepting a set of points $\mathfrak{r}$ in $\pi_{x}$ of measure zero, $\mu\left(\mathfrak{x} ; s_{n}\right)=\kappa^{+}\left(\mathfrak{x}, x, s_{n}^{0}\right)-\kappa^{-}\left(\mathfrak{x}, x, s_{n}^{0}\right)$ for $\mathfrak{x}$ not in $q_{n}$ (see $\left.\S 3.7\right)$. Consequently

$$
\kappa^{+}\left(\mathfrak{x}, x, s_{n}^{0}\right)-\kappa^{-}\left(\mathfrak{x}, x, s_{n}^{0}\right)=\left\{\begin{array}{lll}
0 & \text { for a.e. } & \mathfrak{x} \text { not in } p_{n}^{\prime \prime} ; \\
\operatorname{sgn} J\left(w_{0}, x\right) & \text { for a.e. } \mathfrak{x} \text { in } p_{n}^{\prime} .
\end{array}\right.
$$


From this fact it follows that

$$
\begin{aligned}
& G\left(s_{n}, x\right)=J\left(w_{0}, x\right) \cdot l_{n}^{\prime 2}+\iint_{q_{n}}\left[\kappa^{+}\left(\mathfrak{x}, x, s_{n}^{0}\right)-\kappa^{-}\left(\mathfrak{x}, x, s_{n}^{0}\right)\right], \\
& \left|\iint_{q_{n}}\left[\kappa^{+}\left(\mathfrak{x}, x, s_{n}^{0}\right)-\kappa^{-}\left(\mathfrak{x}, x, s_{n}^{0}\right)\right]\right| \\
& \quad \leqq \iint_{q_{n}} \kappa\left(\mathfrak{x}, x, s_{n}^{0}\right) \leqq \iint_{q_{n}} c\left(\mathfrak{x}, x, s_{n}^{0}\right)+\iint\left[\kappa\left(\mathfrak{x}, x, s_{n}^{0}\right)-c\left(\mathfrak{x}, x, s_{n}^{0}\right)\right] \\
& \quad \leqq\left|J\left(w_{0}, x\right)\right| \cdot 8 m\left(s_{n}\right) l_{n}^{2} / k+G\left(s_{n}, x, \kappa\right)-g\left(s_{n}, x, \kappa\right) .
\end{aligned}
$$

Since $w_{0}$ is not in $E^{3}$ it is true that $D\left(w_{0}, x, \kappa\right)=d\left(w_{0}, x, \kappa\right)$. Because $m\left(s_{n}\right)$ converges to zero as $n$ tends to infinity, it follows from (3) that

$$
\lim _{n \rightarrow \infty} \iint_{q_{n}}\left[\kappa^{+}\left(\mathfrak{x}, x, s_{n}^{0}\right)-\kappa^{-}\left(\mathfrak{x}, x, s_{n}^{0}\right)\right] / l_{n}^{2}=0 .
$$

Since $w_{0}$ is not in $E^{4}$, one now obtains from (2) the relation $D\left(w_{0}, x\right)=J\left(w_{0}, x\right)$, as asserted.

Case (ii). Assume that $J\left(w_{0}, x\right)$ is zero. There exists a sequence of oriented squares $s_{n}$ as described in case (i). Let $r_{n}$ be the rectangle in $\pi_{x}$ associated with $s_{n}$ in the manner described in [6; IV.3.40]; its side lengths are $2^{1 / 2}\left[K+m\left(s_{n}\right)\right] l_{n}$ and $2^{1 / 2} m\left(s_{n}\right) l_{n}$ respectively, and it contains the image of the boundary of $s_{n}$ under $T_{x}$ in its interior. Now $\mu\left(\mathfrak{x}, s_{n}\right)=0$ for $\mathfrak{x}$ not in $r_{n}$, and it follows that $\kappa^{+}\left(\mathfrak{x}, x, s_{n}^{0}\right)-\kappa^{-}\left(\mathfrak{x}, x, s_{n}^{0}\right)=0$ for almost every $\mathfrak{x}$ not in $r_{n}$ by the reasoning used in case $(i)$. Thus

$$
\begin{aligned}
\left|G\left(s_{n}, x\right)\right| & =\iint\left[\kappa^{+}\left(\mathfrak{x}, x, s_{n}^{0}\right)-\kappa^{-}\left(\mathfrak{x}, x, s_{n}^{0}\right)\right] \mid \\
& \leqq \iint_{r_{n}} \kappa\left(\mathfrak{x}, x, s_{n}^{0}\right) \\
& \leqq \iint_{r_{n}} c\left(\mathfrak{x}, x, s_{n}^{0}\right)+\iint\left[\kappa\left(\mathfrak{x}, x, s_{n}^{0}\right)-c\left(\mathfrak{x}, x, s_{n}^{0}\right)\right] \\
& \leqq 2\left[K+m\left(\dot{s_{n}}\right)\right] m\left(s_{n}\right) l_{n}^{2}+G\left(s_{n}, x, \kappa\right)-g\left(s_{n}, x, \kappa\right) .
\end{aligned}
$$

Reasoning as in case (i), one concludes that $D\left(w_{0}, x\right)=0=J\left(w_{0}, x\right)$. Thus relation (1) is established.

4.2. From the preceding theorem one easily obtains the following result.

CoRollary. Let $t: \Re \rightarrow E_{2}$ be a continuous plane transformation which is $e B V$ in $\Re^{0}$ [6; IV.4.1]. Assume that the ordinary Jacobian $J(w, t)$ exists almost everywhere in $\Re^{0}$. Then $J(w, t)=J_{e}(w, t)$ almost everywhere in $\Re^{0}$. 
This constitutes an improvement of the theorem in [6; IV.3.42], where a stronger assumption is placed upon the transformation $t$. In that theorem, however, the domain of definition for $t$ is taken to be a bounded connected open set instead of a bounded simply connected Jordan region $\Re$. The methods used in the proof of the preceding theorem apply at once to this case.

4.3. Under the assumption of the above corollary [ $5 ;$ II.1.2; $4, \S 7]$ Cesari has shown that the ordinary Jacobian equals his generalized Jacobian almost everywhere. However, he has not discussed uniformity features such as those described for our generalized Jacobian in the theorem in 4.1.

\section{BIBLIOGRAPHY}

1. S. Saks, Theory of the integral, Warsaw, 1937.

2. T. Rado and P. Reichelderfer, $A$ theory of absolutely continuous transformations in the plane, Trans. Amer. Math. Soc. vol. 49 (1941) pp. 258-307.

3. L. Cesari, Caratterizzazione analitica delle superficie continue di area finita secondo Lebesgue, Annali della R. Scuola Normale Superiore di Pisa, Serie II vol. 10 (1941) pp. 253-294, vol. 11 (1942) pp. 1-42.

4. - Suifondamenti geometrici dell' integrale classico per l'area delle superficie in forma parametrica, Atti della Reale Accademia d'Italia vol. 13 (1943) pp. 1323-1481.

5. Tibor Rado, Two-dimensional concepts of bounded variation and absolute continuity, Duke Math. J. vol. 14 (1947) pp. 587-608. 1948.

6. - Length and area, Amer. Math. Soc. Colloquium Publications, vol. 30, New York,

7. H. Federer, Essential multiplicity and Lebesgue area, Proc. Nat. Acad. Sci. U.S.A. vol. 34 (1948) pp. 611-616.

8. L. Cesari, Proprieta tangenziali delle superficie continue, Comment. Math. Helv. vol. 22 (1949) pp. 1-16.

9. Tibor Rado, Convergence in area, Duke Math. J. vol. 16 (1949) pp. 61-71.

10. Paul V. Reichelderfer, Law of transformation for essential generalized jacobians, Duke Math. J. vol. 16 (1949) pp. 73-83.

The Ohio State University, Columbus, Ohio. 